\title{
The effect of continuous positive airway pressure treatment on P-wave dispersion and intrinsicoid deflection time in severe obstructive sleep apnea syndrome
}

\author{
Sabri Seyis ${ }^{1}$, Adnan Kazım Usalan ${ }^{2}$
}

\begin{abstract}
Sabri S, Adnan KU. The effect of continuous positive airway pressure treatment on P-wave dispersion and intrinsicoid deflection time in severe obstructive sleep apnea syndrome. Curr Res Cardiol 2017;4(4):61-64.

OBJECTIVE: This study aims to evaluate the effects of continuous positive airway pressure (CPAP) treatment on intrinsicoid deflection time $(\mathrm{R}$ wave peak time) and $\mathrm{P}$ wave discrimination in severe obstructive sleep apnea syndrome (OSAS).
\end{abstract}

METHODS: Fifty-five patients with severe OSAS (apnea-hypopnea index $\geq$ 30) and a healthy control group were included. Baseline electrocardiography (ECG), echocardiography (ECHO), and polysomnography parameters were recorded. All patients received CPAP treatment. ECG and ECHO echocardiography tests were repeated after one-year of CPAP therapy.

RESULTS: A total of 55 OSAS patients (36 M, 19 F) with a mean age of
$56.9 \pm 7.6$ years and 54 healthy controls $(31 \mathrm{M}, 23 \mathrm{~F})$ with a mean age of 56.6 \pm 5.7 years were included. There was a significant improvement in terms of intrinsicoid deflection time in lead V1 $(76.2 \pm 8.3$ vs. $59.8 \pm 7.2)$ and lead V6 $(58.5 \pm 8.0$ vs. $49.8 \pm 7.1)$, and P-wave dispersion $(95.2 \pm 12.8$ vs. $39.8 \pm 6.4)$ after one-year CPAP treatment $(\mathrm{p}<0.001)$. Cardiac volumes and diameters improved significantly.

CONCLUSION: CPAP treatment significantly decreases intrinsicoid deflection time in leads V1 and V6, and improves P-wave dispersion. The results of this study indicate that long-term CPAP treatment had a strong protective effect on right or left cardiac functions in severe OSAS patients without any cardiac and pulmonary disease.

KeyWords: CPAP; Intrinsicoid deflection time; OSAS; P-wave dispersion; Polysomnography
$\mathrm{O}$ bstructive sleep apnea syndrome (OSAS) is a common sleep disorder. It is caused by temporary collapse of the upper airway, which results in nocturnal hypoxia and sleep disturbance $(1,2)$. Many vascular diseases including hypertension, coronary heart disease, heart failure, cardiac arrhythmias, and stroke have been found to be associated with OSAS (3). Cardiovascular morbidity and mortality have been reported as high as $70 \%$ in patients with OSAS (4).

Many studies have shown that OSAS is associated with changes in cardiac structure such as myocardial hypertrophy or increased left ventricular mass, which can widen the QRS complex on the electrocardiogram (ECG) (5-7). On the other hand, prolonged QRS duration is an independent predictor of mortality and cardiovascular diseases (e.g., hypertension and heart failure) in OSAS patients. It has also been shown that severe OSAS patients (AHI $\geq 30$ ) have a four-fold higher frequency of atrial fibrillation (AF) (8). Arrhythmia and its improvement with continuous positive airway pressure (CPAP) therapy in OSAS patients have been reported as well (8). However, the relationship between OSAS and intrinsicoid deflection (ID) (i.e., R-wave peak time) is unknown.

Continuous positive airway pressure is used as first line treatment modality in patients with severe OSAS (9). Regular use of CPAP may lead to reduction in cardiovascular risk in patients with heart failure. A reduction of blood pressure, an increase in left ventricular ejection fraction, and a decrease in left ventricular hypertrophia, and an improvement in cardiovascular function have been demonstrated with echocardiographic (ECHO) findings (10).

Intrinsicoid deflection time is defined as the interval measured from the earliest ventricular activation to the peak of the $R$ wave. This measure represents the time for excitation to spread from the endocardial to epicardial surface of the left ventricle (11). Prolongation of the ID time is frequently associated with diastolic dysfunction. In right ventricular hypertrophy, a prolonged ID time is frequently detected in leads V1 and V2 whereas it is not prolonged in lead V6 (11). Hence, we hypothesized that severe OSAS is associated with prolongation of ID time and P-wave discrimination (PWD), which might be helpful in clarifying the right ventricular hypertrophy and pulmonary hypertension in OSAS patients. Therefore, the aim of this study was to evaluate the impacts of CPAP treatment on ID time and PWD in OSAS patients.

\section{METHODS}

Study design and participants

This prospective was conducted by the Departments of Cardiology and Chest Disease at the Istinye University Liv Hospital and the Medicalpark Hospital. The study protocol was approved by institutional ethics committee. All participants were informed about the study and written consent was obtained before the study. The patients who underwent polysomnography (PSG) at the sleep laboratory with a prediagnosis of OSAS were evaluated and those with severe OSAS (apnea- hypopnea index [AHI] $\geq 30$ events/h) were included in the study. According to the results of polysomnographic study, CPAP treatment was recommended to patients with severe OSAS. All patients underwent physical examination and detailed medical history was taken. Age, gender, body mass index (BMI), resting heart rate, systolic and diastolic blood pressure, presence of hypertension and AHI scores were recorded in all participants. Each patient was assessed with PSG, ECHO, and ECG. After one year of regular CPAP treatment, ECG and ECHO measurements were repeated. Baseline and post-intervention data was compared.

Patients with known right or left bundle branch block, atrial fibrillation, heart valve disorders, advanced heart failure, permanent pacemaker, advanced lung disease, and those using on antiarrhythmic drugs were excluded.

\section{Polysomnography}

All referred subjects with suspected OSAS were subjected to a onenight PSG at the sleep laboratory using the Nox A1 PSG System (Nox Medical, Reykjavik, Iceland). All patients were evaluated by the same physician certified in sleep medicine. Physiological variables evaluated during PSG included; electroencephalogram, electro-oculogram, electromyogram, electrocardiogram, oral and nasal air flow, respiratory effort (thoracoabdominal), body position, and blood oxygen saturation $\left(\mathrm{SpO}_{2}\right)$ (mean and minimum $\mathrm{SpO}_{2}$ ). ECG and anterior tibial electromyography were used to monitor for cardiac arrhythmias and periodic limb movements, respectively. The test was performed according to specific criteria for the definition of sleep stages by trained technicians who were unaware of the patient characteristics.

${ }^{1}$ Department of Cardiology, Istinye University Liv Hospital, Istanbul, Turkey, ${ }^{2}$ Department of Cardiology, Medical Park Tarsus Hospital, Tarsus, Mersin, Turkey

Correspondence: Dr Sabri Seyis, Department of Cardiology, Istinye University Liv Hospital Istanbul, Turkey, Telephone 90533 2472009, e-mail sabriseyis@yahoo.com Received: October 23, 2017, Accepted: November 14, 2017, Published: November 16, 2017

OPEN $\bigcirc$ Access

This open-access article is distributed under the terms of the Creative Commons Attribution Non-Commercial License (CC BY-NC) (http:// creativecommons.org/licenses/by-nc/4.0/), which permits reuse, distribution and reproduction of the article, provided that the original work is properly cited and the reuse is restricted to noncommercial purposes. For commercial reuse, contact reprints@pulsus.com 
Severity of the OSAS was defined according to Apnea-Hypopnea Index $(\mathrm{AHI})$ as mild (AHI $=5$ to 15 events $/ \mathrm{h}$ ), moderate $(\mathrm{AHI}=15$ to 30 events $/ \mathrm{h}$, and severe (AHI>30 events/h). A hypopnea event was defined as a reduction in airflow of at least $50 \%$ or a reduction in the $\mathrm{SpO}_{2}$ of at least $4 \%$ (12).

\section{Echocardiography}

In patients with OSAS, echocardiograms were obtained both before and after CPAP treatment. Echocardiographic parameters were assessed with twodimensional and M-mode cardiovascular ultrasound system (GE-Vingmed Vivid 7 system; Germany). The procedure was performed in the left lateral decubitus position with noninvasive monitoring. Parasternal long-axis and short-axis views and apical views were acquired as standard transthoracic windows. The Simpson method was applied for determining the left ventricular ejection fraction by echocardiography. $\mathrm{ECHO}$ was performed by an experienced cardiologist who was blinded to patient data.

\section{Electrocardiography}

Heart rate, blood pressure, and 12-lead ECG (Cardiovit AT-102 Plus, Schiller, Switzerland) were recorded at rest in the supine position. ECG was performed at the morning hours (between 8:00 and 10:00 AM). ECG measurements of intrinsicoid deflection time in lead V1, in lead V6, and PWD were assessed by an experienced cardiologist who was blinded to the study. The mean of three readings was obtained for further analyses.

The ID on ECG was defined as the time from the onset of the earliest Q to the peak of the $R$ wave ( $R$ wave if the $Q$ wave was absent) (Figure 1).

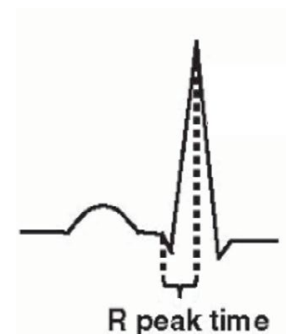

Intrinsicoid
Deflection

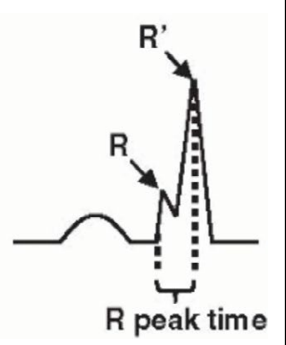

Figure 1) The image showing intrinsicoid deflection time (horizontal distance between the onset of $Q$ and $R$ peak time)

On surface ECG, the P-wave duration was measured from the beginning and to the end of the P-wave. The beginning of the $\mathrm{P}$-wave was defined as the starting point from the baseline for the positive waveform, and as the point of first downward departure from baseline for negative waveforms. The return to the baseline was considered to be the end of the P-wave. The difference between the maximum and minimum P-wave duration was calculated and this difference was defined as $\mathrm{P}-$ wave dispersion ( $\mathrm{PWD}=\mathrm{Pmax}-\mathrm{Pmin})$.

\section{Statistical analysis}

The data were analyzed with SPSS 21.0 for Windows software (SPSS Inc., Chicago, IL, USA). The continuous variables were reported as mean + standard deviation and categorical variables as count (percentage). Kolmogorov-Smirnov test was used to test the normality of distribution. The $\mathrm{t}$-test was used to compare independent groups and the paired t-test was used for within group comparisons. Chi-square test was used to analyze categorical variables. A value of $\mathrm{p}<0.05$ was regarded as statistically significant.

\section{RESULTS}

A total of 55 OSAS patients ( $36 \mathrm{M}, 19 \mathrm{~F})$ with a mean age of $56.9 \pm 7.6$ years and 54 healthy controls $(31 \mathrm{M}, 23 \mathrm{~F})$ with a mean age of $56.6 \pm 5.7$ years were included. Baseline characteristics and echocardiographic data of the participants are summarized in Table 1.

\section{TABLE 1}

Baseline characteristics of the groups

\begin{tabular}{|c|c|c|c|c|}
\hline \multicolumn{2}{|l|}{ Parameters } & $\begin{array}{l}\text { Study } \\
\text { group }\end{array}$ & Control group & $p$ \\
\hline \multicolumn{2}{|l|}{ Age (years) } & $56.9 \pm 7.6$ & $56.6 \pm 5.7$ & 0.841 \\
\hline \multirow{2}{*}{ Sex } & Female & $19(34.5)$ & $23(42.5)$ & 0.388 \\
\hline & Male & $36(65.4)$ & $31(57.4)$ & \\
\hline \multicolumn{2}{|c|}{ Body mass index $\left(\mathrm{kg} / \mathrm{m}^{2}\right)$} & $29.1 \pm 3.9$ & $27.6 \pm 3.1$ & 0.027 \\
\hline \multicolumn{2}{|c|}{ Hypertension (\%) } & $23(41.8)$ & $16(29.6)$ & 0.184 \\
\hline
\end{tabular}

\begin{tabular}{|c|c|c|c|c|}
\hline \multicolumn{2}{|c|}{ Heart rate pulse (min) } & $67.2 \pm 7.0$ & $66.9 \pm 5.9$ & 0.85 \\
\hline \multicolumn{2}{|c|}{ Systolic blood pressure $(\mathrm{mmHg})$} & $131.9 \pm 7.9$ & $126.7 \pm 6.8$ & $<0.001$ \\
\hline \multicolumn{2}{|c|}{ Diastolic blood pressure $(\mathrm{mmHg})$} & $77.5 \pm 6.4$ & $73.8 \pm 6.1$ & 0.003 \\
\hline \multirow{3}{*}{$\begin{array}{l}\text { Electrocardiography } \\
\text { characteristics }\end{array}$} & $\begin{array}{c}\text { Intrinsicoid } \\
\text { deflection in } \\
\text { V1 (ms) }\end{array}$ & $76.2 \pm 8.3$ & $28.0 \pm 4.1$ & $<0.001$ \\
\hline & $\begin{array}{l}\text { Intrinsicoid } \\
\text { deflection in } \\
\text { V6 (ms) }\end{array}$ & $58.5 \pm 8.0$ & $37.8 \pm 4.8$ & $<0.001$ \\
\hline & $\begin{array}{l}\text { P-wave } \\
\text { dispersion } \\
\text { (ms) }\end{array}$ & $95.2 \pm 12.8$ & $39.8 \pm 6.4$ & $<0.001$ \\
\hline
\end{tabular}

Data are presented as mean \pm SD or $n(\%)$.

Chi-square test and t-test were used for comparison.

Bold values show statistically significant $p$ values $(p<0.05)$

There was no statistically significant difference between the groups with respect to the age, gender, presence of hypertension, and heart rate pulse. However, a significant difference was observed between the groups regarding the BMI, ECG findings, and blood pressure parameters. OSAS patients have higher BMI levels compared with the control group ( $29.1 \pm 3.9$ vs 27.6 $\left.\pm 3.1 \mathrm{~kg} / \mathrm{m}^{2}\right)(\mathrm{p}=0.027)$. The ID in leads V1 (ID-V1) and V6 (ID-V6), and PDW values were significantly longer in the OSAS group than in the control group (all $\mathrm{p}<0.001$ ). Baseline and post- treatment echocardiography and electrocardiography findings were compared in Table 2.

\section{TABLE 2}

Baseline and at 1-year follow-up characteristics of the study groups

\begin{tabular}{|c|c|c|c|}
\hline \multirow[b]{3}{*}{ Polysomnography characteristics } & \multicolumn{2}{|c|}{ Study group $(n=55)$} & \multirow[b]{2}{*}{ p-value } \\
\hline & Baseline & At $1^{\text {st }}$ year & \\
\hline & & & \\
\hline Apnea/Hypopnea Index (AHI) & $50.6 \pm 11.3$ & $21.5 \pm 4.6$ & $<0.001$ \\
\hline Mean $\mathrm{O}_{2}$ saturation (\%) & $93.0 \pm 1.4$ & $96.7 \pm 1.1$ & $<0.001$ \\
\hline \multicolumn{4}{|l|}{ Basic characteristics } \\
\hline Heart rate pulse (min) & $67.2 \pm 7.0$ & $64.4 \pm 6.8$ & $<0.001$ \\
\hline Systolic blood pressure $(\mathrm{mmHg})$ & $131.9 \pm 7.9$ & $127.0 \pm 6.8$ & $<0.001$ \\
\hline Diastolic blood pressure $(\mathrm{mmHg})$ & $77.5 \pm 6.4$ & $75.6 \pm 5.4$ & $<0.001$ \\
\hline \multicolumn{4}{|l|}{ Echocardiography characteristics } \\
\hline Ejection fraction (\%) & $61.4 \pm 3.3$ & $61.7 \pm 3.2$ & 0.008 \\
\hline E/A ratio & $0.9 \pm 0.0$ & $0.8 \pm 0.0$ & $<0.001$ \\
\hline LVEDD (mm) & $52.0 \pm 3.6$ & $50.8 \pm 3.5$ & $<0.001$ \\
\hline LVESD (mm) & $37.8 \pm 2.7$ & $37.3 \pm 2.6$ & $<0.001$ \\
\hline IVSD (mm) & $1.2 \pm 0.1$ & $1.16 \pm 0.0$ & $<0.001$ \\
\hline LVPW thickness (mm) & $1.0 \pm 0.0$ & $0.9 \pm 0.0$ & 0.044 \\
\hline \multicolumn{4}{|l|}{ Electrocardiography characteristics } \\
\hline ID in V1 (ms) & $76.2 \pm 8.3$ & $59.8 \pm 7.2$ & $<0.001$ \\
\hline ID in V6 (ms) & $58.5 \pm 8.0$ & $49.8 \pm 7.1$ & $<0.001$ \\
\hline PWD (ms) & $95.2 \pm 12.8$ & $77.8 \pm 11.7$ & $<0.001$ \\
\hline \multicolumn{4}{|l|}{ Cardiac Volumes } \\
\hline End Diastolik Volume (2 chamber) & $119.3 \pm 9.5$ & $126.3 \pm 8.4$ & $<0.001$ \\
\hline End Diastolik Volume (4 chamber) & $124.3 \pm 8.3$ & $129.7 \pm 7.2$ & $<0.001$ \\
\hline End Sistolik Volume (2 chamber) & $45.7 \pm 4.8$ & $48.1 \pm 4.7$ & $<0.001$ \\
\hline End Sistolik Volume (4 chamber) & $47.7 \pm 4.7$ & $49.4 \pm 4.8$ & $<0.001$ \\
\hline
\end{tabular}

Data are presented as mean \pm SD. AHI: Apnea/Hypopnea Index; LVEDD Left Ventricular End Diastolic Diameter; LVESD: Left Ventricular End Sistolic Diameter; LVPW: Left Ventricular Posterior Wall; IVSD: Interventricular Septum Thickness at End-diastole; E/A ratio: Ratio of early and lately mitral flow velocity; PWD: P-wave dispersion; ID: Intrinsicoid Deflection (R-peak time) Paired t-test was used for data analysis. Bold values show statistically significant $P$-values $(p<0.05)$

A significant improvement was observed in terms of PSG, ECHO, and ECG findings (all $\mathrm{p}<0.05)$.

\section{DISCUSSION}

In this study, we have evaluated the impact of one-year CPAP treatment on ECG and ECHO parameters in patients with severe OSAS. According to our results, we have four main findings. First, ID time in leads V1 and V6 significantly prolonged in severe OSAS. Second, patients with severe OSAS had increased PWD. Third, ID time and PWD significantly improved with CPAP treatment. Finally, CPAP treatment improves left ventricular functions and geometries. 
In OSAS, acute physiologic stress occurs during the episodes of asphyxia, including arterial oxygen desaturation, surges in sympathetic activity, and acute hypertension. These cycles may occur hundreds of times a night in a patient with moderate-to-severe OSAS $(13,14)$. Hence, both ventricles are exposed to hemodynamic stress. Systemic or pulmonary arterial pressures, or both, increase during sleep with sleep-related apneas. During apnea periods, the large negative intrathoracic pressures are created during inspiratory efforts, which increases transmural pressure of myocardium, thus increasing afterload. Increased venous return may also produce an increase in preload and pulmonary congestion. Moreover, hypoxemia reduces oxygen delivery to the myocardium, which may induce angina or arrhythmias $(15,16)$. Lastly, frequent arousals from sleep due to respiratory events leads to sympathetic activity and a subsequent increase in catecholamine levels $(17,18)$. Consequently, the adverse events of repetitive episodes of increased afterload on the heart during sleep may persist during the day.

CPAP treatment causes a significant reduction in nocturnal sympathetic activity and blunts blood pressure increments during sleep. Sympathetic nerve responses to CPAP treatment seem to depend on the duration of therapy (19). In a study by Marrone et al., high levels of catecholamines were not reduced after one night of CPAP treatment in OSAS patients (20). After one month of CPAP treatment, a variable effect on sympathetic activity was evident $(15,21)$. Up to two years of CPAP treatment, however, it was reported that a significant reduction in both plasma norepinephrine levels and catecholamine excretion were detected (22).

The effect of CPAP on daytime blood pressure is controversial. While some studies report a reduction in blood pressure, others do not (21-23). A linear relationship between the severity of OSAS and hypertension was recently reported. Besides the higher AHI scores were associated with increased risk of hypertension (24). CPAP treatment reduces the sympathetic nervous system activation associated with apneas (19). Previous studies have already shown the favorable effects of CPAP treatment on blood pressure in OSAS patients $(24,25)$. Likewise, in the present study, compared with the baseline measurements, the CPAP treatment achieved a significantly greater decrease in diastolic and systolic blood pressure, and these reductions were observed in non-hypertensive patients. We believe that our results are noteworthy because prompt intervention with CPAP therapy in OSAS patients might be protective against hypertension. Similarly, heart rate significantly reduced after CPAP treatment. This fact could be attributed to the beneficial effects of CPAP treatment on sympathetic changes.

The ID time represents the time of spreading of excitation from the endocardial surfaces to the epicardial surface of the left ventricle (11). Prolongation of ID shows a delay in ventricular activation and indicates impaired myocardial function. It is frequently associated with diastolic dysfunction. In right ventricular hypertrophy, a prolonged ID time is frequently detected in leads V1 and V2 whereas it is not prolonged in lead V6. On the other hand, PWD is a predictive of AF and OSAS patients have increased risk of arrhythmia. From this point of view, we can say that OSAS patients have increased risk of cardiovascular events (5-8). In our study, mean ID time in Lead V1 was $76.2 \pm 8.3$, and it was significantly improved by longterm CPAP usage $(59.8 \pm 7.2)$. Additionally, P-wave discrimination was 95.2 \pm 12.8 and significantly improved $(77.8 \pm 11.7)$ with CPAP treatment. Herein, we highlight the potential role of CPAP therapy in bypassing the risk factors for cardiovascular events.

The beneficial effect of CPAP on cardiac functions such as improved myocardial oxygen delivery, decreased sympathetic activity, left ventricular transmural pressure, and afterload have been reported in many studies (26). Furthermore, Alchanatis et al. studied 15 OSAS patients, and determined left ventricular diastolic dysfunction and increased diastolic blood pressure, each of which improved following 3 to 4 months of nasal CPAP treatment (27). Besides, arrhythmia reduction and improved pulmonary hypertension have also been reported with CPAP (27). Similarly, Cloward et al. report that left ventricular hypertrophy was detected in high frequency in subjects with severe OSAS and regressed after 6 months of nasal CPAP treatment (28). In this study, Clowart et al. report that $92 \%$ of the 25 subjects had structural abnormalities on echocardiography, and $88 \%$ of subjects had left ventricular hypertrophy, $64 \%$ had left atrial enlargement, $48 \%$ had right atrial enlargement, and $16 \%$ had right ventricular hypertrophy. In addition, ejection fraction was within normal limits in all patients (28). In their study, although the primary measures of left ventricle hypertrophy obtained from echocardiograms were interventricular septal thickness at diastole and left ventricle posterior wall thickness, a regression after six months of CPAP treatment was observed. However, no significant change was found in left arterial enlargement or right arterial enlargement following 6 months of CPAP.
The association of OSAS and right heart function is incompatible. The prevalence of right ventricular hypertrophy by ECHO in sleep apnea was up to $71 \%$ (29). In the present study, mean ID time in Lead V1 was $76.2 \pm 8.3$, and significantly improvement was seen by long-term CPAP therapy ( 59.8 \pm 7.2 ). P-wave discrimination was $95.2 \pm 12.8$, and it was also significantly improved $(77.8 \pm 11.7)$ by CPAP treatment. Likewise, our results show that, severe OSAS patients had prolonged ID time in leads V1 and V6, and so PWD, and regressed after one year of CPAP therapy.

Our study has some limitations. First, this study included only severe OSAS patients, not mild to moderate OSAS patients. Lack of evaluation of the right cardiac functions with ECHO is another limitation. Lastly, lack of data as regards to the CPAP compliance is a limitation as well.

\section{CONCLUSION}

In conclusion, long-term CPAP treatment significantly decreases ID time in lead V1 and V6 and also improves PWD in patients with severe OSAS. It may be beneficial to provide a strong protective effect on right and left cardiac functions in severe OSAS patients without any cardiac and pulmonary disease.

\section{FUNDING SOURCES}

This research did not receive any specific grant from funding agencies in the public, commercial, or not-for-profit sectors.

\section{CONFLICTS OF INTEREST}

The authors have no conflicts of interest.

\section{REFERENCES}

1. Dursunoglu N, Dursunoglu D. Obstructive sleep apnea syndrome, endothelial dysfunction and coronary atherosclerosis. Tuberk Toraks 2005;53:299-306.

2. Young T, Palta M, Dempsey J, et al. The occurrence of sleep-disordered breathing among middleaged adults. N Engl J Med 1993;328:1230-5.

3. Fava C, Montagnana M, Favaloro EJ, et al. Obstructive sleep apnea syndrome and cardiovascular diseases. Semin Thromb Hemost 2011;37:280-97.

4. Lee CH, Khoo SM, Tai BC, et al. Obstructive sleep apnea in patients admitted for acute myocardial infarction. Chest 2009;135:1488-95.

5. Morin DP, Oikarinen L, Viitasalo M, et al. QRS duration predicts sudden cardiac death in hypertensive patients undergoing intensive medical therapy: the LIFE study. Eur Heart J 2009;30:2908-14.

6. El-Solh AA, Akinnusi ME, Baddoura FH, et al. Endothelial cell apoptosis in obstructive sleep apnea: a link to endothelial dysfunction. Am J Respir Crit Care Med 2007;175:1186-91.

7. Malhotra A, White DP. Obstructive sleep apnoea. Lancet 2002;360:237-45.

8. Digby GC, Baranchuk A. Sleep apnea and atrial fibrillation; 2012 update. Curr Cardiol Rev 2012;8:265-72.

9. National Institute for Health and Clinical Excellence. Continuous positive airway pressure for obstructive sleep apnoea/hypopnoea syndrome.

10. Dursunoglu N, Dursunoglu D, Çuhadaroğlu C, et al. Acute effects of automated CPAP on blood pressure in the patients with sleep apnea and hypertension. Respiration 2005;72:150-5.

11. O’Neal WT, Qureshi WT, Nazarian S, et al. Electrocardiographic time to intrinsicoid deflection and heart failure: The multi-ethnic study of atherosclerosis. Clin Cardiol 2016;39:531-6.

12. The Report of an American Academy of Sleep Medicine Task Force Sleep related breathing disorders in adults: recommendations for syndrome definition and measurement techniques in clinical research. The Report of an American Academy of Sleep Medicine Task Force. Sleep 1999;22:667-89.

13. Lattimore JD, Celermajer DS, Wilcox I. Obstructive sleep apnea and cardiovascular disease. J Am Coll Cardiol 2003;41:1429-37.

14. Narkiewicz K, Kato M, Phillips BG, et al. Nocturnal continuous positive airway pressure decreases daytime sympathetic traffic in obstructive sleep apnea. Circulation 1999; 100:2332-5. 
15. Stenlof K, Grunstein R, Hedner J, et al. Energy expenditure in obstructive sleep apnea: effects of treatment with continuous positive airway pressure. Am J Physiol 1996;271:E1036-43.

16. Dimsdale JE, Coy T, Ziegler MG, et al. The effect of sleep apnea on plasma and urinary catecholamines. Sleep 1995;18:377-81.

17. Garvey JF, Taylor CT, McNicholas WT. Cardiovascular disease in obstructive sleep apnoea syndrome: the role of intermittent hypoxia and inflammation. Eur Respir J 2009;33:1195-205.

18. Jelic S, Padeletti M, Kawut SM, et al. Inflammation, oxidative stress, and repair capacity of the vascular endothelium in obstructive sleep apnea. Circulation 2008;117:2270-8.

19. Chen L, Scharf SM. Systemic and myocardial hemodynamics during periodic obstructive apneas in sedated pigs. J Appl Physiol 1998;84:1289-98.

20. Marrone O, Riccobono L, Salvaggio A, et al. Catecholamines and blood pressure in obstructive sleep apnea syndrome. Chest 1993;103:722-7.

21. Jennum P, Wildschiodtz G, Christensen NJ, et al. Blood pressure, catecholamines, and pancreatic polypeptide in obstructive sleep apnea with and without nasal continuous positive airway pressure (nCPAP) treatment. Am J Hypertens 1989;2:847-52.

22. Hedner J, Darpo B, Ejnell H, et al. Reduction in sympathetic activity after long-term CPAP treatment in sleep apnoea: cardiovascular implications. Eur Respir J 1995;8:222-9.
23. Suzuki M, Otsuka K, Guilleminault C. Long-term nasal continuous positive airway pressure administration can normalize hypertension in obstructive sleep apnea patients. Sleep 1993;16:545-9.

24. Mokros $Ł$, Kuczyński W, Franczak $Ł$, et al. Morning diastolic blood pressure may be independently associated with severity of obstructive sleep apnea in non-hypertensive patients: A cross-sectional study. J Clin Sleep Med 2017;13:905-10

25. Arnaud C, Dematteis M, Pepin JL, et al. Obstructive sleep apnea, immuno-inflammation, and atherosclerosis. Semin Immunopathol 2009;31:113-25.

26. Dursunoglu N, Dursunoglu D, Ozkurt S, et al. Effects of CPAP on right ventricular myocardial performance index in obstructive sleep apnea patients without hypertension. Respir Res 2006;7:22.

27. Alchanatis M, Paradellis G, Pini $\mathrm{H}$, et al. Left ventricular function in patients with obstructive sleep apnoea syndrome before and after treatment with nasal continuous positive airway pressure. Respiration 2000;67:367-71.

28. Cloward TV, Walker JM, Farney RJ, et al. Left ventricular hypertrophy is a common echocardiographic abnormality in severe obstructive sleep apnea and reverses with nasal continuous positive airway pressure. Chest 2003;124:594-601.

29. Berman EJ, DiBenedetto RJ, Causey DE, et al. Right ventricular hypertrophy detected by echocardiography in patients with newly diagnosed obstructive sleep apnea. Chest 1991;100:347-50. 